\title{
Grand Challenges in Astronomical Instrumentation
}

\author{
Jeff R. Kuhn* \\ Institute for Astronomy, University of Hawaii, Honolulu, HI, United States
}

Keywords: astronomical instrumentation, Daniel K. Inouye Solar Telescope, exoplanet direct imaging, gravitational wave detection, ExoLife Finder, Moore's Law

\section{INTRODUCTION}

How do measuring instruments, that are so fundamental to modern science, shape our knowledge of space around us? Obviously, they provide the building blocks for astrophysical theories, with the biggest leaps in understanding coming from the most powerful instruments, but what makes a powerful instrument? These are not inert parts of the scientific process. They are defined not just by our technology reach but by "prejudice" - presumed understanding only partially bounded by measurements, but not established fact. The form or even existence of dark matter in the universe is one example of such a prejudice. It has launched many instruments aimed at a well-known and venerably incomplete perspective. Astronomical remote sensing instruments project information from other parts of the universe onto a set of local observables. The power of these come from both the scope of the enlightened prejudice that formulated their observables, and the potential to change (or "factualize") this prejudice over the working life of the instrument.

Interesting instrument histories are framed by the creative intangibles that mark their development, and the conclusions that follow from their output data. Evidently great visions flow from enlightened prejudice and evolving technological capabilities. These establish the directions and distance of a reach that is both enabled and limited by peculiar human choices. This essay is one view of how human resources should be used to create new astronomical instrumentation stories.

Some of my perspectives came from an earlier generation. The instrument Bob Dicke, Ken Libbrecht and I built in the 1980's to measure the shape of the Sun was "state-of-the-art" but barely digital. It created datasets that were 256, 16 bit scalars (Dicke et al., 1985). Digitization was enabled by discrete Transistor-Transistor Logic (TTL) electronics and we used an analog motor rotation signal to extract harmonics of the Sun's shape. This astronomical instrument was conceived with a small set of observables and as a narrowly-focused experiment capable of answering limited scientific questions. It yielded datasets that could almost be analyzed with paper and pencil or a desk-top calculator. That old optical telescope-detector system projected information from the Sun onto a single scalar observable-the oblateness of the solar limb.

Then, we were at the doorstep of a new generation of instruments enabled by the possibility of routine and immediate digital observables from the increasing information bandwidth of digital technologies. Film, astronomical plates, and discrete diode arrays were about to give way to direct Charge Coupled Device (CCD) measurements of two-dimensional images, spectra, and polarization signals that were carried by photons and delivered to instruments on the backs of telescopes. Although these early digital instruments were tiny by standards today, I vividly recall the excitement in our lab when Ed Loh (Loh and Wilkinson, 1976) scientifically empowered the $0.6 \mathrm{~m}$ Princeton observatory telescope with this early digital astronomical camera. It was based around just a $100 \times 100$ pixel CCD that could hardly generate $1 \mathrm{MB}$ of data in a full observing session. Yet, it seemed like over-night, the information from an astronomical remote sensing instrument jumped by orders of magnitude. 
The remote sensing instruments of today are barely recognizable in this first CCD imager. Consider, for example, in the later part of 2020, the room-sized visible and infrared imaging spectropolarimeters (Harrington and Sueoka, 2017) that will be deployed at the coude focus of the Daniel K. Inouye Solar Telescope (DKIST) (NSO, 2020; Rimmele, 2020). DKIST will enable the biggest leap in solar observing capability since Galileo improved human visual observations with his $4 \mathrm{~cm}$ telescope. In contrast, these first-light DKIST instruments will generate many terabytes of 3- and even 4-dimensional spectropolarimetry observables each day. This represents an expansion in the information rate from astronomical instruments by 6 orders of magnitude over a 40 year period, or a doubling time in the capacity to create new astronomical information of about 2 years.

New remote sensing instruments are paced by technical advances in optics, photonics, and material or information science. Their output data's information content is accelerating in step with "Moore's Law" and information technology's growth, like it is in other human domains (Wikipedia, 2020). The astronomical instruments being conceived and built today have complexity that we could hardly imagine a generation earlier. A grand challenge in remote sensing instruments that is upon us now, is to find better ways for humans to interpret the vast information outputs implied by Moore's Law growth. I believe that many prejudices will evolve as even our definition of "remote sensing" will change.

One might characterize the current generation of astronomical instruments by their data rates of terabytes per day. For example, the DKIST instruments will average 8 Tbyte/day (Davey et al., 2016; and this is a telescope that observes only one object in the sky) and can produce 50 Tbyte on a busy day. The PanSTARRS (Chambers et al., 2016; IfA, 2020) and the Rubin (LSST, 2020) telescopes survey most of the full-sky and create about 15 terabytes/day.

It seems unlikely that appetites for raw remote sensing information will fall behind instrument technology doubling rates, but this growth will eventually saturate. When instruments are capable of recording the direction, wavelength, and polarization of each photon that passes through the telescope we will have captured the available remote information. At that point, only telescope improvements in wavelength, angular resolution, or aperture can extend our electromagnetic reach into the universe. Proposed instruments on DKIST or Rubin are several orders of magnitude away from this, and will take perhaps 20 years at the current rate to reach this quantum limit.

An immediate challenge for our instrument community is to devise information processing (i.e., "data pipeline") platforms that more directly allow creative access to these ballooning datasets. The data system for the PanSTARRS survey telescope and its gigapixel cameras is a significant fraction of the cost of the telescopes. The data system for the Rubin telescope is $1 / 3$ of the project cost. The vast datasets DKIST will produce depend on a data pipeline that is a large fraction of the operation costs. This trend toward increasingly complex instrument data systems surely will continue. The ability to search and analyze data with trainable machine learning algorithms as part of the instrument system [perhaps in the mode of (Duev et al., 2019)], could help frame and answer scientific questions that we don't even know how to pose with existing astrophysical models. The challenge here may be to build accessible machine learning capabilities directly into the structure of future instruments and their data systems.

Each of the above instrument systems relied on a telescope that was fundamentally integrated into its detectors. For example, the DKIST instruments are effectively spectropolarimeters designed to work with a telescope that is as much a polarimeter and coronagraph as it is a tool for collecting more light. This is no accident but was required by a prejudice-an incomplete vision of how magnetism controls the solar atmosphere. This drives the need for polarization observables. Similarly the PanSTARRS and Rubin observatories' wide-field survey prejudice effectively defined the instruments on the telescope back-ends. In this case questions about yet undetected asteroids and the largescale structure of the Universe and its early history defined the telescope-instrument systems. The physical distinction between telescope and astronomical instrument will further blur as we achieve information bandwidths that obviate their functional differences. Another grand challenge will be to find even better ways to use new technology to fully integrate telescopes and instrument-detectors in order to solve the great remaining remote sensing questions.

One of the grandest challenges ahead is the revelation of life outside the solar system, or the Search for Extraterrestrial Intelligence (SETI). Previous generations, driven by a prejudice for what electromagnetic information may be leaking (or intentionally beamed) from advanced life elsewhere, have recognized this as a data analysis problem in its purest form (Garrett, 2015; SETI Inst, 2020). The current generation now owns the problem in perhaps a more powerful way. They're the first to realize that there are more exoplanets in the galaxy than stars (NASA, 2020a) and have the technological hope of remotely sensing exoplanet life. Solving this is a challenge that will mature some of our most broad-reaching prejudices, like notions about the origins and robustness of life. The problem of directly finding and measuring bio- or techno-signatures from an exoplanet requires exquisitely sensitive measurements of the reflected or emitted light from the orbiting planet. Sorting the few exoplanet photons from the million- to billiontimes more that come directly from the exoplanet host star is the "direct imaging problem." Its solution requires a large telescope and detector system that work as one to capture enough life-signature photons. But it must also achieve extreme coronagraphic performance that distinguishes the exoplanetary photons from the overwhelming stellar flux (Berdyugina and Kuhn, 2019). One proposed solution, called the ExoLife Finder (Kuhn et al., 2018), follows the template of the DKIST and Rubin instruments - to integrate essential characteristics of a light-gathering telescope with the detector. In this case, to detect exoplanet photons before they are scrambled with the stellar light by telescope diffraction and scattering. The essential technology exists now and we should look forward to waking up some morning to the announcement that life, or even advanced life, has been found around a nearby star that is not the Sun. 
This electromagnetic vision of the Universe will soon be fundamentally challenged. The reality of gravitational wave (GW) remote sensing instruments and GW detection has been the most exciting scientific discovery of my lifetime (Abbott et al., 2016). Space-time perturbations yield an unobscured view into the distant universe that may even change our prejudices about the boundaries between quantum mechanics and General Relativity (Croker et al., 2020). The potential of the next generation GW instruments in space (NASA, 2020b) presents a grand challenge that we must find resources to pursue.

Finally, the ultimate challenge that may change even our definition of remote sensing, could combine in-situ DNA sequencing of a distant exolife environment and communication from Earth with interstellar instrument-probes. Yuri Milner's Breakthrough Foundation has started making generationspanning plans to send such probes to our nearest stars (Breakthrough, 2020). It is a grand and speculative vision that must solve some almost impossibly difficult engineering problems, but the payoff to humanity could be priceless.

\section{REFERENCES}

Abbott, B. P., Abbott, R., Abbott, T. D., Abernathy, M. R., Acernese, F., Ackley, K., et al. (2016). GW151226: observation of gravitational waves from a 22-solar-mass binary black hole coalescence. Phys. Rev. Lett. 116:241103. doi: 10.1103/PhysRevLett.116.241103

Berdyugina, S. V., and Kuhn, J. R. (2019). Surface imaging of proxima b and other exoplanets: albedo maps, biosignatures, and technosignatures. Astronomical J. 158:246. doi: 10.3847/1538-3881/ab2df3

Breakthrough (2020). Breakthrough Initiatives. Available online at: https:// breakthroughinitiatives.org/initiative/3 (accessed May 22, 2020).

Chambers, K. C., Magnier, E. A., Metcalfe, N., Flewelling, H. A., Huber, M. E., Waters, C. Z., et al. (2016). The PanSTARRS-1 Surveys. arXiv [Preprint]. arXiv: 1612.05560 .

Croker, K. S., Nishimura, K. A. and Farrah, D. (2020). Implications of symmetry and pressure in friedmann cosmology. II. stellar remnant black hole mass function. Astronomical J. 889:115. doi: 10.3847/1538-4357/ab5aff

Davey, A. R., Reardon, K., Berukoff, S. J., Hays, T., Spiess, D., Watson, F. T. et al. (2016). The DKIST Data Center: Meeting the Data Challenges for Next-Generation, Ground-Based Solar Physics. San Francisco, CA: American Geophysical Union, Fall General Assembly.

Dicke, R. H., Kuhn, J. R., and Libbrecht, K. G. (1985). Oblateness of the Sun in 1983 and relativity. Nature 316:687. doi: 10.1038/316687a0

Duev, D. A., Mahabal, A., Masci, F. J., Graham, M. J., Rusholme, B., Walters, R., et al. (2019). Real-bogus classification for the Zwicky Transient Facility using deep learning. Mon. Not. R. Astron. Soc. 489, 3582-3590. doi: $10.1093 / \mathrm{mnras} / \mathrm{stz} 2357$

Garrett, M. (2015). SETI reloaded: next generation radio telescopes, transients, and cognitive computing. Acta Astron. 113, 8-12. doi: 10.1016/j.actaastro.2015.03.013

Harrington, D. M., and Sueoka, S. R. (2017). Polarization modeling and predictions for Daniel K. Inouye Solar Telescope part 1: telescope and example
Astronomical instrument science is riding an exponential wave of information growth. It will continue to expand our reach into the universe with tools that effectively blur the distinction between telescopes and detectors. Such instrument systems will see well-beyond what mere electromagnetic signals can reveal. Their bounty of knowledge will likely exceed the scientific realm of traditional astrophysical models and challenge our prejudices in fields as diverse as biology to geology. These advances may even have existential value to humanity.

\section{AUTHOR CONTRIBUTIONS}

The author confirms being the sole contributor of this work and has approved it for publication.

\section{ACKNOWLEDGMENTS}

JK acknowledges useful comments on this manuscript from Thomas Rimmele and Ken Chambers.

instrument configurations. J Astron Telescopes Instruments Systems 3:018002. doi: 10.1117/1.JATIS.3.1.018002

IfA (2020). The PanSTARRS Survey. Available online at: http://www.ifa.hawaii.edu/ research/Pan-STARRS.shtml (accessed May 22, 2020).

Kuhn, J. R., Berdyugina, S. V., Capsal, J.-F., Gedig, M., Langlois, M., Moretto, G., et al. (2018). The Exo-Life Finder Telescope (ELF): Design and Beam Synthesis Concepts. (Austin, TX: SPIE). doi: 10.1117/12.2312779

Loh, E. D. and Wilkinson, D. T. (1976). Direct photography with a bare CCD BAAS. Bulletin of the American Astronomical Society. 8:350.

LSST (2020). The Rubin Observatory. Available online at: https://www.lsst.org (accessed May 22, 2020).

NASA (2020a). Exoplanet Exploration. Available online at: https://exoplanets.nasa. gov/ (accessed May 22, 2020).

NASA (2020b). Laser Interferometer Space Antenna. Available online at: https:// lisa.nasa.gov/ (accessed May 22, 2020).

NSO (2020). The Daniel K. Inouye Solar Telescope. Available online at: https://dkist. nso.edu (accessed May 22, 2020).

Rimmele (2020). The Daniel K. Inouye solar telescope. Sol. Phys.

SETI Inst (2020). Available online at: https://seti.org (accessed June 9, 2020)

Wikipedia (2020). Moore's Law. Available online at: https://en.wikipedia.org/wiki/ Moore\%27s_law (accessed May 22, 2020).

Conflict of Interest: The author declares that the research was conducted in the absence of any commercial or financial relationships that could be construed as a potential conflict of interest.

Copyright (C) $2020 \mathrm{Kuhn}$. This is an open-access article distributed under the terms of the Creative Commons Attribution License (CC BY). The use, distribution or reproduction in other forums is permitted, provided the original author(s) and the copyright owner(s) are credited and that the original publication in this journal is cited, in accordance with accepted academic practice. No use, distribution or reproduction is permitted which does not comply with these terms. 